\title{
QuPWM: Feature Extraction Method for Epileptic Spike Classification
}

\author{
Abderrazak Chahid ${ }^{1}$, Member, IEEE, Fahad Albalawi ${ }^{2,1}$, Member, IEEE, Turky Nayef Alotaiby ${ }^{3}$, Majed Hamad \\ Al-Hameed ${ }^{4}$, Saleh Alshebeili, Taous-Meriem Laleg-Kirati ${ }^{1, \star}$, Senior Member, IEEE
}

\begin{abstract}
Epilepsy is a neurological disorder ranked as the second most serious neurological disease known to humanity, after stroke. Inter-ictal spiking is an abnormal neuronal discharge after an epileptic seizure. This abnormal activity can originate from one or more cranial lobes, often travels from one lobe to another, and interferes with normal activity from the affected lobe. The common practice for Inter-ictal spike detection of brain signals is via visual scanning of the recordings, which is a subjective and a very time-consuming task. Motivated by that, this paper focuses on using machine learning for epileptic spikes classification in magnetoencephalography (MEG) signals. First, we used the Position Weight Matrix (PWM) method combined with a uniform quantizer to generate useful features from time domain and frequency domain through a Fast Fourier Transform (FFT) of the framed raw MEG signals. Second, the extracted features are fed to standard classifiers for inter-ictel spikes classification. The proposed technique shows great potential in spike classification and reducing the feature vector size. Specifically, the proposed technique achieved average sensitivity up to $87 \%$ and specificity up to $97 \%$ using 5 -folds cross-validation applied to a balanced dataset. These samples are extracted from nine epileptic subjects using a sliding frame of size 95 samplespoints with a step-size of 8 sample-points.
\end{abstract}

Index Terms-magnetoencephalography (MEG), Position Weight Matrix (PWM), Epileptic spike detection, machine learning

\section{INTRODUCTION}

$\mathbf{E}$ PILEPSY is not a singular disease entity but a variety of dysfunctions reflecting brain disorders or abnormal electrical activities that may strike patients of all ages [1]. An epileptic seizure occurs when a burst of electrical impulses in the brain exceeds its normal limits. These pulses spread to neighboring areas in the brain, which may create an uncontrolled storm of electrical activity sent to body organs depending on the size and location of the affected region (see Fig. 1). Epileptic activity may affect brain areas related to motor functions, causing twitches or convulsion. In particular, epileptic patients may stare blankly for a few seconds during

1 Computer, Electrical and Mathematical Sciences and Engineering Division (CEMSE) King Abdullah University of Science and Technology (KAUST), KSA. E-mail:abderrazak.chahid@kaust.edu.sa, taousmeriem.laleg@kaust.edu.sa

${ }^{2}$ Electrical Engineering Department, Taif University, Taif, 21955, KSA. Email: fahad.albalawi@kaust.edu.sa

${ }^{3}$ King Abdulaziz City for Science and Technology (KACST), Riyadh , KSA. E-mail: totaiby@kacst.edu.sa

4 National Institute of Neuroscience, King Fahad Medical City, Riyadh 11525, KSA.

${ }^{5}$ King Saud University (KSU), Riyadh, KSA. E-mail:dsaleh@ksu.edu.sa

* Corresponding authors e-mails: taousmeriem.laleg@kaust.edu.sa, dsaleh@ksu.edu.sa a seizure, while others have uncontrollable jerking movements of the arms and legs. Among the diagnostic tools for epilepsy is the magnetoencephalography (MEG) [2]. The MEG is a recent functional neuro-imaging technology that measures the magnetic activity of the brain. This new technology uses an array of highly sensitive sensors or magnetometers called superconducting quantum interference devices (SQUIDs). However, as the brain magnetic field is very weak compared to surrounding magnetic sources, the MEG measurement needs a shielded room to isolate the patient from the external magnetic fields such as the magnetic field of the earth or the electronic devices. For Epileptic patients, the MEG is used during two main phases of the treatment [3], [4]: first, localizing the region of the brain, which produces the abnormal electrical activities that cause the neurological disorder. If the localized region is not responsible for a vital function in the brain, such as speech, this region is usually extracted surgically if the medications were not effective for the treatment. Second, assessing surgery outcomes.

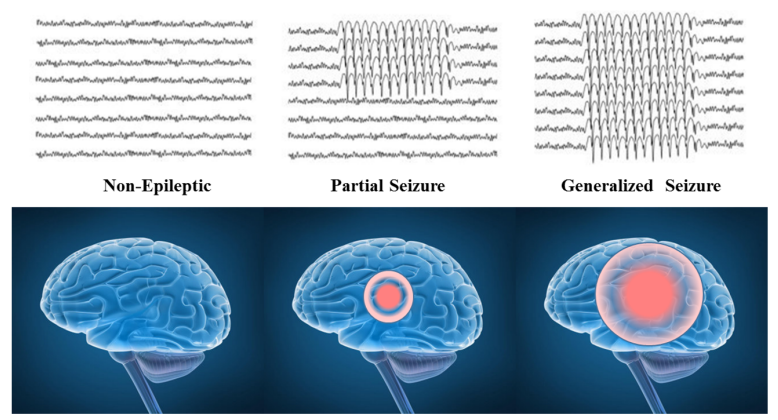

Fig. 1: Illustration of the brain abnormal activities for different types of epileptic seizures [built based on [5] [6]].

Proper diagnosis and treatment of epilepsy is a major public health goal. An essential routine clinical step inaccurate diagnosis and treatment of epilepsy is the identification and detailed examination of interictal epileptiform discharges (IED) from Electroencephalography (EEG) and, where available, MEG recordings. Currently, the examination of MEG and EEG signals in clinical practice are performed largely manually. Visual identification by expert reviewers is the current gold standard and a widely used procedure for detecting IEDs in both MEG and EEG recordings. This type of manual detection allows much flexibility and adaptability to recordings with largely different and varying baseline levels, artifacts, and IEDs. However, it requires visually analyzing large amounts of data, which is time-consuming, produces fatigue, and is prone 
to errors and inconsistencies . For this reason, automated interictal epileptic spike detection in MEG signals has attracted research interest over the last decade. Four methods, to the best of our knowledge, have been proposed for spike detection of the MEG signals in literature [7], [8]. The first method uses the independent component analysis (ICA). The authors used the ICA decomposition to find statistically independent components in multi-sensor/ multi-channel MEG signals to perform spatial filtering of the MEG data and separate interictal spikes from background activity and then apply clustering technique for the detection [9]. The detection is performed using a thresholding technique. The second method is based on the common spatial patterns (CSP) method and linear discriminant analysis (LDA) (CSP-LDA) [10]. The CSP-LDA performs eigenvalue decomposition of the covariance matrices of the input data to find the most discriminative features. LDA classifier is then employed to perform the spikes detection. The third method is the Amplitude Thresholding and Dynamic Time Warping (AT-DTW) proposed in [11]. This method first uses amplitude thresholding to determine the most likely spiky segments. For the spike detection, the method employs dynamic time warping. The maximum specificity and sensitivity reported in the aforementioned detection methods are $95.8 \%$, and $92.4 \%$, respectively. A more recent detection method has been proposed in [12], where the decomposition of the signal into squared eigenfunctions of the Schrodinger operator was used for detection [13]. This method uses the largest negative eigenfunction in the absolute value of the discrete spectrum of the Schrödinger Operator as a feature with the Random Forest (RF) classifier.

In this work, we propose a novel feature generation method for a multi-channel MEG signal, which improves patientindependent inter-ictal spike detection. This method is based on the combination of the Position Weight Matrix (PWM) method and the uniform quantization scheme. We name this approach QuPWM. This method takes advantage of the efficiency of the PWM method, which is usually used for DNA sequences classification. QuPWM shows great potential in improving the accuracy of the inter-ictal spike detection models.

The paper is organized as follows. Section II includes a description of the MEG dataset and the proposed features extraction process methods based on the PWM method and the used quantization schemes in Section II. Section III presents the obtained results using two MEG experiment: first, healthy versus epileptic patients. Second, using spiky versus non-spiky sample in epileptic patients. Section IV presents a discussion of the findings, where a conducted sensitivity analysis on the proposed feature extraction method is analyzed with respect to the frame length, number of subjects, and using different dataset such as EEG. Finally, Sections V summarizes our concluding remarks.

\section{Materials AND Methods}

The proposed methodology consists of three main steps, as shown in Fig. 2. First, the MEG recordings are split into segments using framing technique. For instance, the first frames of the 24 channels are concatenated to form the first sample, and so on. Then the samples are converted into sequences using a uniform quantization scheme. Second, the quantified samples are mapped to extract two types of PWMbased features. Finally, the classification model SVM is used for spike detection. These three steps are explained in the following sections II-B and II-C.

\section{A. Experimental data acquisition and analysis}

MEG data were recorded in a shielded room at National Neural Institute- King Fahad Medical City (NNI-KFMC), Riyadh, Saudi Arabia, with an Elekta Neuromag system. As the MEG signals are much weaker than normal environmental magnetic noise, the shielded room blocks the majority of environmental magnetic fields so that the magnetic fields generated by the brain can be accurately detected. Elekta Neuromag head system (helmet) contains 102 magnetometer and 204 gradiometer sensors. These sensors are further categorized according to the different brain regions. Clinically, the brain is divided into eight regions; left temporal (LT), right temporal (RT), left frontal (LF), right frontal (RF), left parietal (LP), right parietal (RP), left occipital (LO), and right occipital (RO). Each element of the Elekta Neuromag system is comprised of three sensors, one magnetometer, and two gradiometers. Magnetic brain activity was recorded at a sampling frequency of $1 \mathrm{kHz}$. MEG data was filtered by tSSS (Spatiotemporal signal space separation) method [14]. The data were then offline band-pass filtered $1-50 \mathrm{~Hz}$ for visual inspection. A total of 18 MEG data segments, each of 15 minutes duration and 24 channels, were taken from eight epileptic patients and eight healthy patients. These segments are analyzed by specialized neurologists from NNI, KFMC, Riyadh. The neurologists marked the MEG spikes locations, in different brain regions, by visual inspection. The number of sessions, total number of spikes, and the average spike duration in these recordings are described in table I as follows:

TABLE I: Detailed description of the MEG dataset.

\begin{tabular}{|c|c|c|c|}
\hline Subject ID & \#Sessions & \#Spikes & Average spikes duration \\
\hline E001 & 3 & 24 & $53.96 \mathrm{~ms}$ \\
\hline E002 & 3 & 17 & $266.24 \mathrm{~ms}$ \\
\hline E003 & 2 & 5 & $81.60 \mathrm{~ms}$ \\
\hline E004 & 3 & 23 & $89.22 \mathrm{~ms}$ \\
\hline E005 & 3 & 11 & $86.27 \mathrm{~ms}$ \\
\hline E006 & 3 & 30 & $79.83 \mathrm{~ms}$ \\
\hline E007 & 3 & 29 & $90.68 \mathrm{~ms}$ \\
\hline E008 & 3 & 16 & $75.69 \mathrm{~ms}$ \\
\hline E009 & 3 & 12 & $40.83 \mathrm{~ms}$ \\
\hline
\end{tabular}

As mentioned earlier, there are 306 sensors to cover the whole head. These sensors are further marked according to the brain regions. Written informed consent was signed by each participant or responsible adult before they participated 


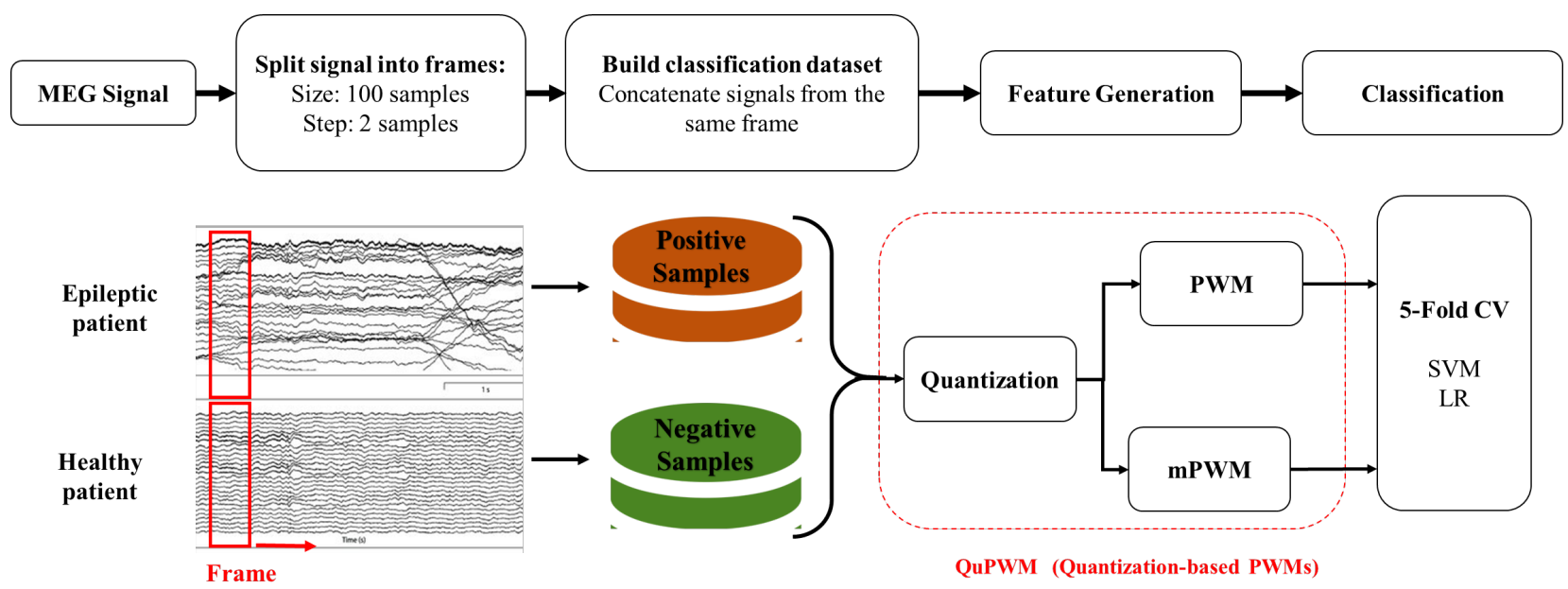

Fig. 2: The experiment 1 framework: MEG records pre-processing, feature generation and classification models.

in the study. The study was conducted in accordance with the approval of the Institutional Review Board at KFMC (IRB log number: 15-086, 2015).

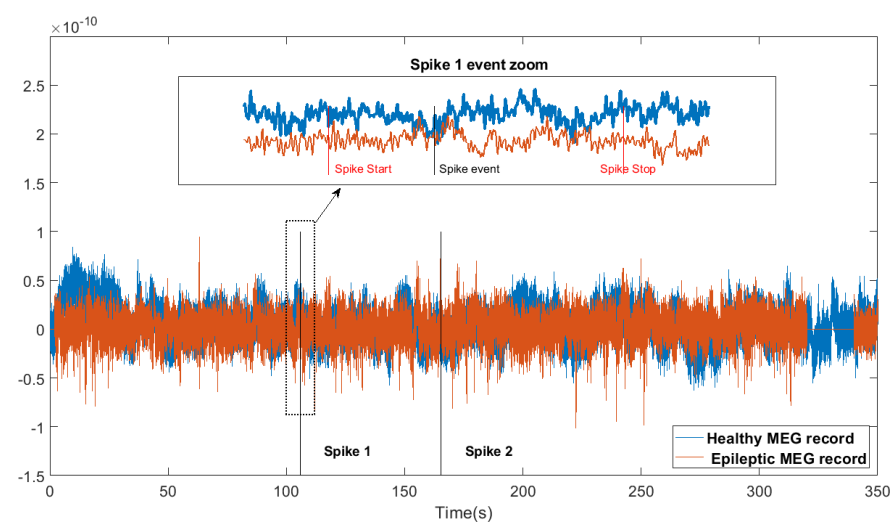

Fig. 3: Example of healthy and epileptic subject MEG recording. Zoomed plot shows a segment where an epileptic spike event happens in epileptic subject (orange) compared to a healthy subject (black).

\section{B. Multi-channels $M E G$ signal pre-processing}

Multi-channels MEG pre-processing aims essentially to prepare classification samples. The pre-processing consists of three steps:

- signal Framing: build equitized samples that combine sliding windows or frames from all the 24 MEG channels. These combined frames will represent the raw data samples used as inputs to the classification, which will be quantified in the next step.

- FFT projection: with respect to the QuPWM features, the input signals should be preferably aligned in order to get better classification performance. Motivated by that, we consider applying frequency domain projection through a Fast Fourier Transform (FFT) to the framed MEG signals.

- signal Quantization: convert the real-values samples to sequences of letters. This step is needed to make the samples suitable for the used feature generation method explained in Sections II-C1 and II-C2.

1) MEG signal Framing: The multi-channel MEG signals are segmented into frames using sliding frames using a sliding window of size 100 sample-points with a step of 2 samplepoints, as shown in Figures 2 and 3. For instance, the first 100 sample-points of all channels are concatenated together to form the first sample of size 2400 sample-points of the input raw-data. A binary label or class is assigned to each sample as follows:

- Positive class: represent samples that have epileptic spike events occurring in MEG records of the epileptic patients.

- Negative class: represent samples from uniformly distributed time-locations in MEG records of the healthy patients.

After signal framing, the positive and negative samples will be quantified using a uniform quantizer, as explained in the next section.

2) FFT projection to frequency domain: The input framed signal are transformed to the frequency domain using FFT transform of $x(n)$ to frequency domain as $X(k)$ defined as follows:

$$
X(k)=\frac{1}{N} \sum_{0}^{N} x(n) \mathrm{e}^{\frac{2 \pi k n}{N}}
$$

where $N$ is the number of sampling-points of the signal $x(n)$.

In the frequency domain version of the signal, each position/sampling-point represents a specific frequency component in all signals forming alignment of the input framed signals. Which allows all input signal to be suitable for position weight matrix method.

3) $M E G$ signal quantization: In this work we employed the PWM method for feature extraction as explained in Section II-C1. However, as the PWM method deals only with 
sequences, the input MEG samples should be converted into sequences, as shown in Fig. 4. For this reason, we used a uniform quantization scheme. The quantization is utilized to convert the real-valued signal $X$ to a sequence $Q$ of different levels $q_{1}, q_{2}, \ldots, q_{M}$ defined as follows [15], [16]:

$$
Q(n)=q_{i} \quad \text { where } \quad i=1,2, \ldots, M
$$

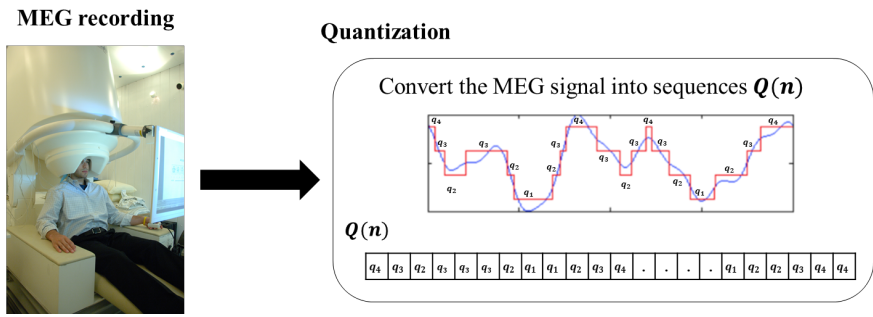

Fig. 4: Example of MEG signal quantization using four levels.

In this classification, the quantization is used to reduce the weight matrices size compared to applying the PWM directly to real-valued signal, because every real value has to be considered as a level in its own. The quantizer will regroup different values of the real-valued input signal into one single level $q_{i}$ ( see Fig. 4). The number of levels $M$ will depend on the probability density of the dataset, as shown in Fig 5. Therefore, two major parameters of the quantization scheme are defined: the desired number of levels $M$ and the resolution $\mathbf{r}$. For an appropriate choice of these parameters, the probability distribution of the signal is analyzed for both classes.

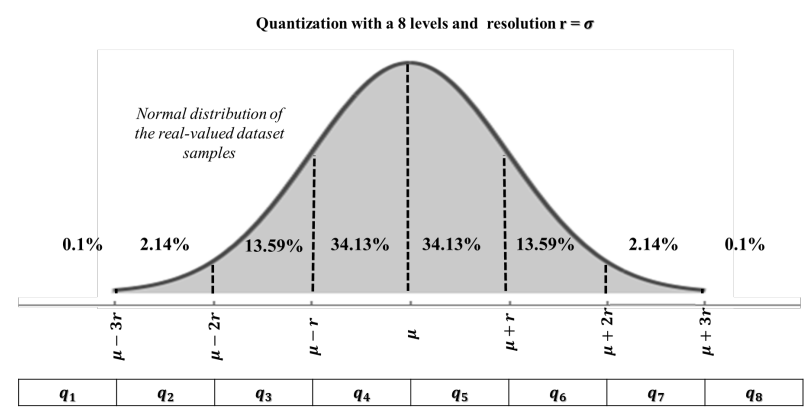

Fig. 5: The quantization of the real-valued sequence with a resolution $r=\sigma$ and $M=8$. The percentage values reflect the significance of probability of each interval.

Indeed, it is known from probability theory and mathematical statistics that the value of a random variable is considered rare to happen if it lies far from its mean $\mu$ by more than $3 \sigma$. This observation is called the 3-sigma rule [17] [18]. Therefore, only the most significant variability or randomness characterizing of a Gaussian random variable $X$ is considered, while the other values are neglected. This fact is observed if :

$$
|X(n)-\mu| \leq 3 \sigma
$$

where $\mu$ and $\sigma$ are defined to be the mean and standard deviation of the Gaussian random variable $X$.
The standard rule is used to define the quantization scheme, as shown in Fig. 5. It is important to note that the probability distribution of $X$ varies from subject to another, which affects the quantization scheme. Therefore and in order to unify the quantization scheme, the mean $\mu$ and standard deviation $\sigma$ are defined as the average mean and standard deviation of all subjects as follows:

$$
\mu=\frac{1}{N_{s}} \sum_{n=1}^{N_{s}} \mu_{n} \quad \text { and } \quad \sigma=\frac{1}{N_{s}} \sum_{n=1}^{N_{s}} \sigma_{n},
$$

where $N_{s}$ is the number of subjects. $\mu_{n}$ and $\sigma_{n}$ are the mean and the standard deviation of the data values of the $n^{t h}$ subject.

Since the 3-sigma rule indicates that most of the information of a Gaussian random variable is located within $3 \sigma+\mu$, the quantization resolution is set to be equal to the standard deviation $\sigma$ as shown in Fig. 5 and explained in detail in Algorithm 1.

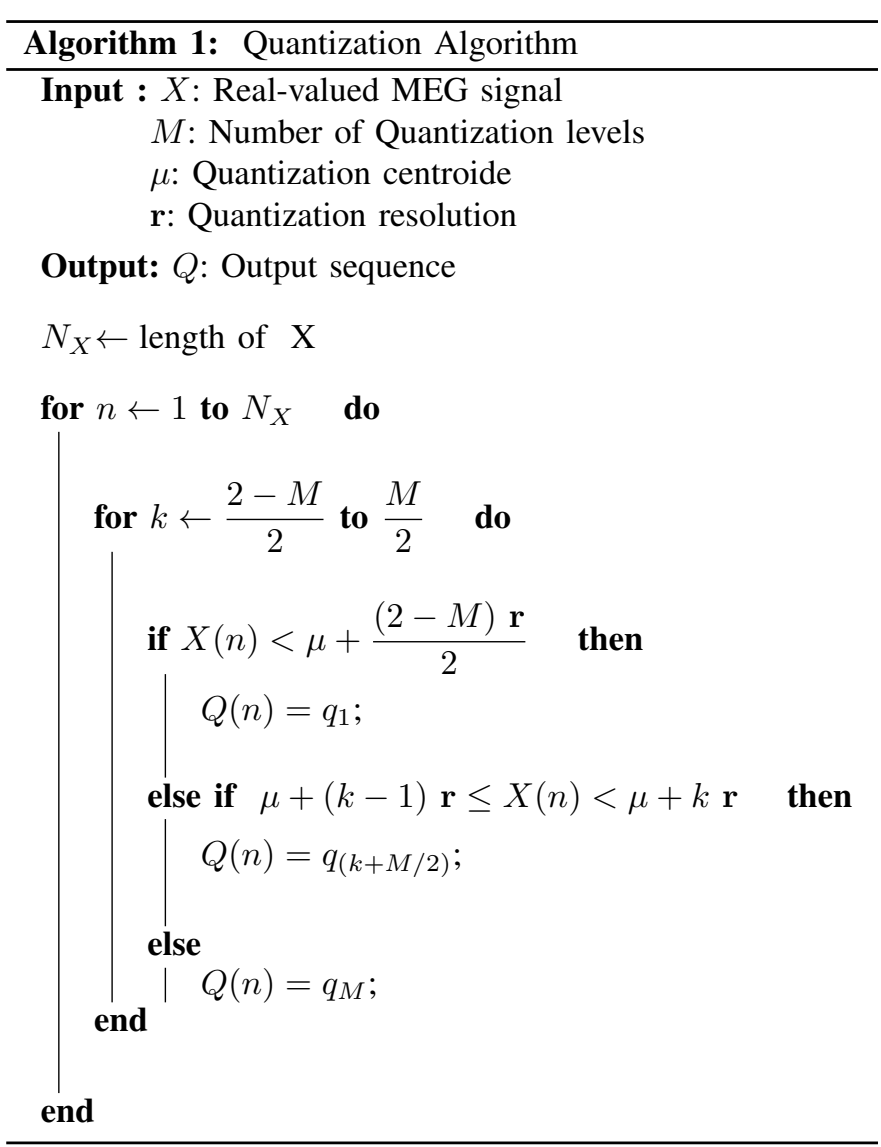

\section{QuPWM-based features generation}

The proposed Quantization-based position weight matrix (QuPWM) feature generation method is based on combining the position weight matrix (PWM) method with Quantization. This method uses two approaches to generate PWM features, as explained in the next section. 
1) Standard PWM-based features : A position weight matrix (PWM), also known as a position-specific weight matrix (PSWM) or position-specific scoring matrix (PSSM), has been presented for the first time by Gary in [19]. This method is widely used technique for motifs characterization and discovery in biological sequences such as DNA/mRNA [20]-[24]. It showed high potential in sequences characterization and motifs extracting with remarkable binary classification accuracy. For MEG signals, we adapted the PWM-based method to extract relevant features after signal quantization methods. The PWM is based on building two matrices, $P W M^{+}$derived from the positive training set and the second $P W M^{-}$represents the negative set. The PWM matrices indicate the significance of each position within input sequences for each class. For binary classification, the two matrices $P W M^{+}$and $\mathrm{PWM}^{-}$ are defined as follows:

$$
P W M^{+}\left[i, q_{j}\right]=\sum_{s=1}^{N_{s}^{+}} \xi\left(Q_{s}^{+}(i), q_{j}\right)
$$

and

$$
P W M^{-}\left[i, q_{j}\right]=\sum_{s=1}^{N_{s}^{-}} \xi\left(Q_{s}^{-}(i), q_{j}\right)
$$

where $N_{s}^{+}$and $N_{s}^{-}$are the total number of sequences in the positive and negative classes. The $\xi(a, b)$ function is defined as follows:

$$
\xi(a, b)= \begin{cases}1 & \text { if the } a=b \\ 0 & \text { elsewhere }\end{cases}
$$

Table II shows an example of a positive matrix $P W M^{+}$. Each column of the matrix represents the frequency of a specific letter $q_{i}$ in a specific position $n$ among all positive sequences.

TABLE II: Example of a position-wight matrix $P W M^{+}$ containing the frequencies of five levels $\left[q_{1}, q_{2}, \ldots, q_{5}\right]$.

\begin{tabular}{cccccc}
\hline$n^{t h}$ & \multicolumn{5}{c}{ Quantization levels } \\
\cline { 2 - 6 } position & $q_{1}$ & $q_{2}$ & $q_{3}$ & $q_{4}$ & $q_{5}$ \\
\hline 1 & 1104 & 257 & 331 & 384 & 372 \\
2 & 1209 & 213 & 271 & 291 & 324 \\
3 & 1217 & 245 & 298 & 336 & 336 \\
4 & 987 & 263 & 309 & 409 & 474 \\
5 & 941 & 253 & 383 & 442 & 532 \\
6 & 1273 & 243 & 308 & 317 & 367 \\
7 & 1494 & 208 & 243 & 261 & 245 \\
8 & 1301 & 264 & 299 & 364 & 388 \\
9 & 61 & 101 & 306 & 829 & 1331 \\
10 & 144 & 88 & 266 & 599 & 1133 \\
.. &.. &.. &.. &.. &.. \\
\hline
\end{tabular}

Then, these two PWMs are used to generate two scores representing the extracted features. The two scores $\operatorname{Score}^{+}(\mathbf{Q})$

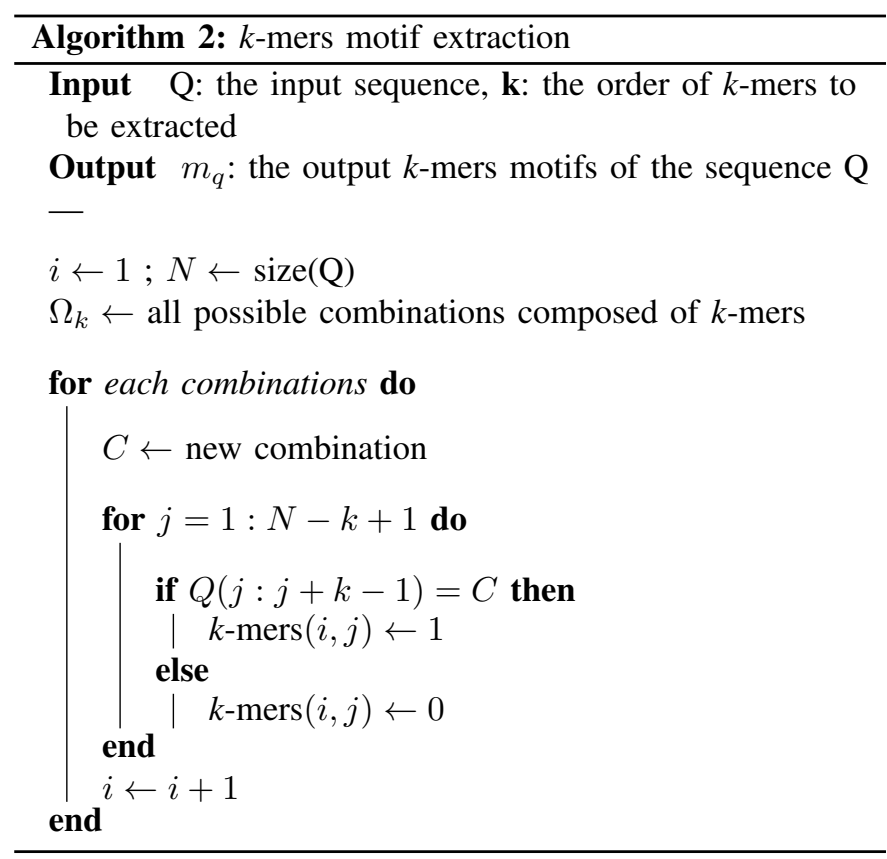

and $\operatorname{Score}^{-}(\mathbf{Q})$ represent the two probabilities of a given sequence to be in the positive or negative class. In other words, for a given sequence $Q$ to be positive, the score induced by $P W M^{+}$should be greater than the score induced by $P W M^{-}$. The two scores $\operatorname{Score}^{+}(\mathbf{Q})$ and $\operatorname{Score}^{-}(\mathbf{Q})$ of a sequence $Q$ are defined as as follows:

$$
\operatorname{Score}^{+}(\mathbf{Q})=\frac{\sum_{n=1}^{N} P W M^{+}[n, Q(n)]}{\sum_{j=1}^{M} P W M^{+}\left[n, q_{j}\right]}
$$

and

$$
\operatorname{Score}^{-}(\mathbf{Q})=\frac{\sum_{n=1}^{N} P W M^{-}[n, Q(n)]}{\sum_{j=1}^{M} P W M^{-}\left[n, q_{j}\right]}
$$

where $N$ is the number of sample-points in the data sample/signal $Q$.

It is very important to mention that the PWMs should be reconstructed only from the training dataset in order not to violate the classification rules.

2) Motif-based PWM features: In order to extract more advanced patterns, we adopted a new approach which deals with binary sequences, extracted from the original sequence, that represent the presence of a specific motif of one letter or more in the original sequence, as shown in Fig. 6. We called this approach the motif-based Position Weight Matrix (mPWM). The main idea is to decompose every sequence into binary sequences reflecting a specific pattern of levels in this sequence such as :mono-mers $\left(q_{1}, q_{2}, q_{3}\right.$, etc $)$, di-mers $\left(q_{1} q_{1}\right.$ , $q_{1} q_{2}, q_{1} q_{3}$, etc $)$, tri-mers $\left(q_{1} q_{1} q_{1}, q_{1} q_{2} q_{3}, q_{3} q_{3} q_{3}\right.$, etc $)$. For instance, a sequence of 3 levels $q_{1}, q_{2}, q_{3}$ will give: 3 possible mono-mers, $3 \times 3=9$ possible di-mers combinations, and $3 \times 3 \times 3=27$ possible tri-mers combinations. Fig. 6 shows the different $k$-mer encodings. For instance, the binary sequence of the mono-mers $m_{q_{1}}$ is defined as follows: 


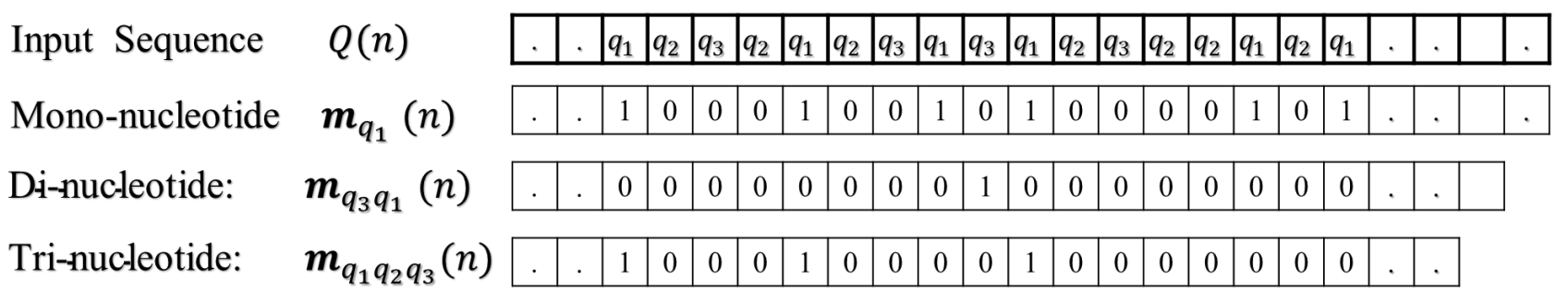

Fig. 6: Examples of three binary sequences corresponding to mono-mers, di-mers and tri-mers, respectively.

$$
m_{q_{1}}(n)= \begin{cases}1 & \text { if } Q(n)=q_{1} \\ 0 & \text { elsewhere }\end{cases}
$$

Similarly, the binary sequence of the di-mers $m_{q_{1} q_{3}}$ is defined as follows:

$$
m_{q_{1} q_{3}}(n)= \begin{cases}1 & \text { if } Q(n)=q_{1} \text { and } Q(n+1)=q_{3} \\ 0 & \text { elsewhere }\end{cases}
$$

The binary mapping of $k$-mers motif extraction is summarized in Algorithm 2.

TABLE III: Example of position-wight matrix $P W M_{\mathbf{q}}^{+}$containing the frequencies of di-mer motif.

\begin{tabular}{cccccccccc}
\hline$n^{\text {th }}$ & \multicolumn{8}{c}{ Di-mer motifs $(\mathbf{k = 2})$} \\
\cline { 2 - 10 } position & $q_{1} q_{1}$ & $q_{1} q_{2}$ & $q_{1} q_{3}$ & $q_{1} q_{4}$ & $q_{2} q_{1}$ & $q_{2} q_{2}$ & $q_{2} q_{3}$ & $q_{2} q_{4}$ &.. \\
\hline 1 & 15 & 13 & 11 & 14 & 17 & 13 & 11 & 8 &.. \\
2 & 10 & 10 & 12 & 5 & 9 & 11 & 16 & 6 &.. \\
3 & 0 & 0 & 0 & 0 & 0 & 0 & 0 & 0 &.. \\
4 & 0 & 0 & 0 & 0 & 0 & 0 & 0 & 0 &.. \\
5 & 8 & 10 & 8 & 12 & 7 & 14 & 3 & 12 &.. \\
6 & 298 & 290 & 310 & 294 & 303 & 293 & 322 & 299 &.. \\
7 & 93 & 93 & 101 & 112 & 94 & 109 & 89 & 116 &.. \\
8 & 0 & 0 & 0 & 0 & 0 & 0 & 0 & 0 &.. \\
.. &.. &.. &.. &.. &.. &.. &.. &.. &.. \\
\hline
\end{tabular}

Similarly to the standard PWM, the binary sequences $m_{q}$, representing the $k$-mer motifs $\mathbf{q} \in \Omega_{k}$ and which is defined in Eq. 14, is used to reconstruct multiple pair of PWM matrices $P W M_{q}^{+}$(see Table III) and $P W M_{q}^{-}$defined as follows:

$$
P W M_{\mathbf{q}}^{+}(n)=\sum_{s=1}^{N_{s}^{+}} \xi\left(m_{q, s}^{+}(n), 1\right)
$$

and

$$
P W M_{\mathbf{q}}^{-}(n)=\sum_{s=1}^{N_{s}^{-}} \xi\left(m_{q, s}^{-}(n), 0\right)
$$

where $\mathbf{q} \in \Omega$, where $\Omega=\Omega_{1} \cup \Omega_{2} \cup \ldots \cup \Omega_{k}$ and $\Omega_{k}$ represents the set of the possible $k$-mers combination defined as:

$$
\Omega= \begin{cases}\Omega_{1}=q_{1}, q_{2}, q_{3}, \ldots & \text { 'mono-mers' } \\ \Omega_{2}=q_{1} q_{1}, q_{1} q_{2}, q_{1} q_{3}, \ldots & \text { 'di-mers' } \\ \Omega_{3}=q_{1} q_{1} q_{1}, q_{1} q_{2} q_{3}, q_{3} q_{3} q_{3}, \ldots & \text { 'tri-mers' } \\ \ldots & \end{cases}
$$

$m_{q, s}$ denotes the motif $m_{q}$ extracted from the $s^{t} h$ sequence $Q_{s} . N_{s}^{+}$and $N_{s}^{-}$are the total number of sequences in the positive and negative classes. $\xi$ function is defined in Eq. 7.

Similarly, the two scores $S_{\text {core }}+\left(m_{q}\right)$ and Score $^{-}\left(m_{q}\right)$ of a $k$-mer $m_{q}$, extracted from a sequence $Q$, are defined as as follows:

$$
\operatorname{Score}^{+}\left(m_{q}\right)=\frac{\sum_{n=1}^{N} m_{q}(n) P W M_{\mathbf{q}}^{+}(n)}{\sum_{n=1}^{N} P W M_{\mathbf{q}}^{+}(n)}
$$

and

$$
\operatorname{Score}^{-}\left(m_{q}\right)=\frac{\sum_{n=1}^{N}\left(1-m_{q}(n)\right) P W M_{\mathbf{q}}^{-}(n)}{\sum_{n=1}^{N} P W M_{\mathbf{q}}^{-}(n)}
$$

where $\mathbf{q} \in \Omega_{1} \cup \Omega_{2} \cup \ldots \cup \Omega_{k}$ as defined in Eq. 14, where $\mathbf{k}$ is the order of the extracted k-mers in the QuPWM features.

\section{Classification models}

The input MEG raw data after the pre-processing step has 1734 spiky samples and 1734 healthy samples from different MEG test sessions of eight healthy and eight epileptic patients. The quantified samples are used to generate QuPWM features and then fed to different classification models: K-Nearest Neighbors, Support Vector Machine (SVM), Decision Tree (DT), Random Forest (RF), Multi-layer Perceptron (MLP) and Naive Bayes (NB). The 5-folds cross-validation (CV) process is used, where the data is randomly split into five folds. Each time, four folds are used for training and the remaining fold for testing. The CV performance is measured by average accuracy, sensitivity, and specificity of all five folds. The operation is repeated 10 times in order to study the robustness of the classification model. Finally, the best model which achieves the highest average accuracy is reported.

\section{RESULTS}

For epileptic patient treatment, the patient needs to take the MEG test to ensure that the abnormal brain activities 


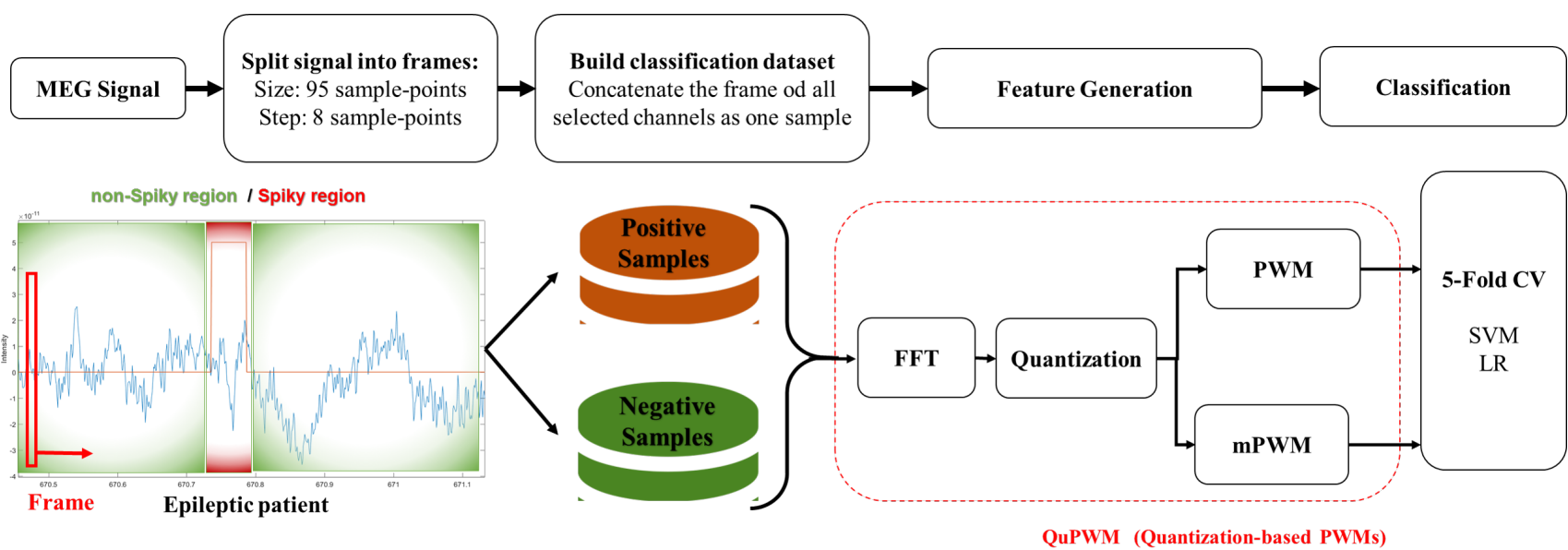

Fig. 7: Experiment 2 framework using frequency domain projection.

are absent. Up to now, the visual assessment is the "only" way to assess the MEG signals. Indeed, there is no efficient computer-based aid to analyze MEG signals. Therefore, there is a need for developing machine learning-based algorithms to assist clinicians in quickly and efficiently detecting and predicting abnormalities in MEG signals. In this paper, we will show the utility of the proposed feature generation method for biomedical signal classification. Therefore, the QuPWM features will be used for two different classification experiments. The details of each classification experiments and the obtained results are described in the coming sections III-A and III-B, respectively.

\section{A. Experiments 1 :Spike classification for healthy VS epileptic patients}

For this classification, the positive samples are extracted from the spiky region in eight epileptic patients, and the negative samples are from eight healthy patients using MEG signals. This classification investigates the ability of the QuPWM method to learn from normal brain activity in healthy patients to predict inter-actal spike.

The framework of this experiment is illustrated in Fig. 2. The epileptic spike prediction performance of this classification is shown in Table IV.

TABLE IV: Feature-size reduction comparison of the of proposed PWM features

\begin{tabular}{|c|c|c|c|c|c|c|}
\hline Method & Parameters & Classifier & Feature size & Accuracy & Sensitivity & Specificity \\
\hline Raw data & - & - & 2400 & 60.06 & 78.27 & 41.84 \\
\hline$P W M$ & $\mathrm{M}=8, \mathbf{r}=0.18$ & SVM & $\mathbf{2}$ & 92.10 & 91.10 & 93.10 \\
\hline$m P W M$ & $\begin{array}{l}\mathrm{M}=10, \mathbf{r}=0.15 \\
\mathbf{k}=2\end{array}$ & SVM & 220 & $\mathbf{9 8 . 2 3}$ & $\mathbf{9 8 . 0 7}$ & $\mathbf{9 8 . 3 9}$ \\
\hline
\end{tabular}

\section{B. Experiments 2 : Spike classification for epileptic patients}

For this classification, the positive samples are extracted from spiky regions, while the negative samples are from the non-spiky regions of nine different epileptic patients using
MEG signals. This classification is much harder than the previous one, and it challenges the QuPWM ability to distinguish between spiky and non-spiky regions in epileptic patients. The QuPWM feature from the time domain does not achieve good performance because the extracted spiky and non-spiky samples are quite similar in the time domain, as shown in Fig. 8.

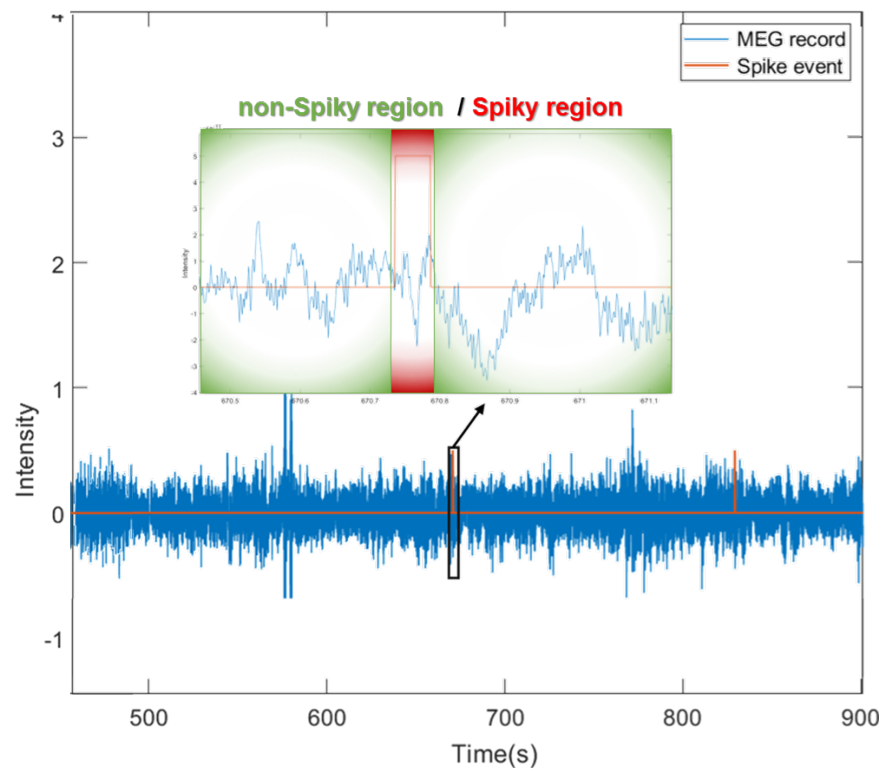

Fig. 8: MEG splitting and dataset preparation for the classification experiments 2 .

Therefore, the frequency domain is used through a ShortTime Fourier Transform of the MEG signals before applying the QuPWM feature extraction method, as shown in Fig. 7. By doing so, the QuPWM starts extracting frequency-related scores describing the discriminative patterns in the MEG signals spectrum.

\section{Subject-independent classification}

For epileptic spikes detection, it is important to build subject-independent models that can provide higher specificity 
TABLE V: Comparison of the proposed methods with existing methods reported recently in [11]

\begin{tabular}{|c|c|c|c|c|c|}
\hline Method & \#Subjects & Feature & Accuracy & Sensitivity & Specificity \\
\hline ICA [9] & 4 & $\begin{array}{l}\text { Independent Components } \\
\text { Analysis }\end{array}$ & - & 86.91 & 81.19 \\
\hline CSP-LDA [10] & 20 & $\begin{array}{l}\text { Common Spatial Patterns and } \\
\text { Linear Discriminant Analysis }\end{array}$ & - & 86.14 & 90.38 \\
\hline AT-DTW [11] & 30 & $\begin{array}{l}\text { Amplitude Thresholding and } \\
\text { Dynamic Time Warping }\end{array}$ & - & 92.45 & 95.81 \\
\hline QuPWM-SVM & 9 & $\begin{array}{l}\text { di- mers motif-based Position Weight Matrix } \\
\text { SVM classifer }\end{array}$ & 92.48 & 87.20 & 97.76 \\
\hline
\end{tabular}

and sensitivity for any number of subjects. In order to study the effect of the number of subjects on the detection performance, we studied the combination of randomly combined subjects using an SVM classifier and different frame-lengths $L=\{80,90,95,100,110\}$. The QuPWM-based features show a constant performance for most of the combinations using the optimal frame-size 95 sample-points, as shown in Table VI. This is because the QuPWM-based features extract the common patterns within the dataset regardless of its size. However, for very big size data, this remark might not hold.

\section{Comparison with existing methods}

Several detection methods have been proposed for spikes detection using MEG signals. The proposed features generation method is compared to the reported performance of some recent works [11]. Table V shows that the proposed feature generation methodology improves the classification sensitivity and specificity. However, it is worth to be mentioned that the proposed method only works for a balanced dataset due to the limitation of the feature extraction method while the other reported methods were applied to unbalanced datasets.

\section{DISCUSSION}

\section{A. Comparison of the PWM-based features}

Each of the utilized QuPWM approaches captures a different property of the spikes characteristic. In order to study how these features perform and compare their performances, the different PWM approaches have been tested on the same dataset, using the same classifier, which is the SVM. The obtained results are shown in Tables IV and VII. The results show that the performance of the PWM method can be improved significantly using the mPWM feature. However, the mPWM method needs a larger memory and longer time. This limitation can be addressed using parallelization programming and feature selection to select the most important motif to extract.

\section{B. Choice of the frame length $L$}

As explained in Section II-A, the training and testing data are extracted from the MEG records using a sliding window or frame of size $L$. The choice of the frame-size is motivated by the statistical properties of the spikes time-duration for the different eight subjects. Most of the spikes last almost 95ms, as shown in Fig. 9. Therefore, the frame should be long enough to capture the common characteristics of the different spikes. In order to study how sensitive are the proposed feature generation methods, we performed a sensitivity analysis to study the effect of the frame-length on the classification. The obtained average performance in 5-folds cross-validation using logistic regression classifier with a set of frame-lengths $L=\{80,90,95,100,110\}$ is presented in Table VII. It shows that a frame-length 95 sample-points $=95 \mathrm{~ms}$ gives better prediction accuracy. Therefore, the optimal frame-length should be around the mean spike duration for all subjects.

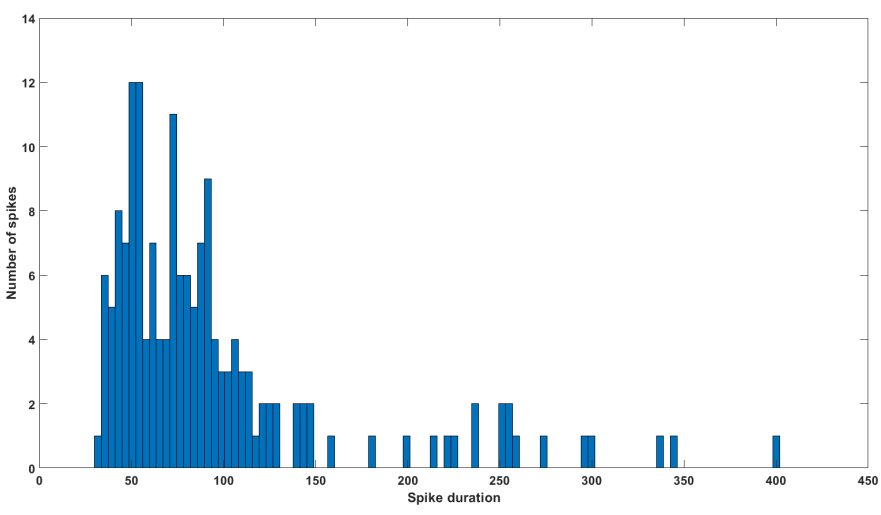

Fig. 9: The statistical properties of the epileptic spikes duration for eight epileptic subjects.

\section{The effect of classifier type on the performance}

In order to study the impact of the classifier on the obtained results, several classifiers were used for spike classification. Specifically, we applied several classifiers: SVM, DT, NB, KNN, and LR. Table VIII shows that the SVM classifier achieves better results using the radial basis function kernel or RBF kernel.

\section{Further validation using EEG classification}

In order to investigate the usefulness of the proposed method, the QuPMW method is applied to Epileptic Seizure 
TABLE VI: . The 5-Folds cross-validation performance for different subjects using LR classifier (mPWM: $M=6, r=$ $0.33, k=1)$.

\begin{tabular}{|c|c|c|c|c|c|c|c|c|}
\hline Subject ID & Feature size & Accuracy & Sensitivity & Specificity & Precision & Gmean & F1score & AUC \\
\hline$E 001$ & 12 & 92.18 & 88.24 & 96.12 & 95.79 & 92.09 & 91.86 & 0.96 \\
\hline$E 002$ & 12 & 92.10 & 88.25 & 95.94 & 95.60 & 92.02 & 91.78 & 0.96 \\
\hline$E 003$ & 12 & 92.10 & 88.18 & 96.02 & 95.68 & 92.01 & 91.77 & 0.96 \\
\hline$E 004$ & 12 & 92.08 & 88.20 & 95.95 & 95.61 & 91.99 & 91.76 & 0.96 \\
\hline$E 005$ & 12 & 92.10 & 88.15 & 96.05 & 95.72 & 92.01 & 91.77 & 0.96 \\
\hline$E 006$ & 12 & 92.04 & 88.20 & 95.87 & 95.52 & 91.96 & 91.72 & 0.96 \\
\hline$E 007$ & 12 & 92.15 & 88.27 & 96.03 & 95.70 & 92.07 & 91.83 & 0.96 \\
\hline$E 008$ & 12 & 92.11 & 88.33 & 95.89 & 95.55 & 92.03 & 91.80 & 0.96 \\
\hline$E 009$ & 12 & 92.22 & 88.37 & 96.06 & 95.73 & 92.14 & 91.90 & 0.96 \\
\hline
\end{tabular}

\begin{tabular}{|l|l|l|l|l|l|l|l|l|}
\hline All subjects & 12 & 92.10 & 88.40 & 96.17 & 95.68 & 92.09 & 91.60 & 0.96 \\
\hline
\end{tabular}

TABLE VII: The effect of fame length $L$ on 5-Folds cross-validation performance.

\begin{tabular}{|c|c|c|c|c|c|c|c|c|c|}
\hline \#Subjects & Size & $\mathbf{L}$ & Step & Method & Parameters & Feature size & Accuracy & Sensitivity & Specificity \\
\hline \multirow{10}{*}{9} & 21800 & 80 & \multirow{5}{*}{8} & \multirow{5}{*}{ PWM } & \multirow{5}{*}{$\mathrm{M}=5, \mathrm{k}=0.25$} & \multirow{5}{*}{2} & 86.39 & 77.67 & 95.12 \\
\hline & 23142 & 90 & & & & & 87.53 & 79.08 & 95.99 \\
\hline & 23754 & 95 & & & & & 87.49 & 78.88 & 96.11 \\
\hline & 24270 & 100 & & & & & 87.19 & 77.85 & 96.52 \\
\hline & 25122 & 110 & & & & & 87.07 & 78.24 & 95.89 \\
\hline & 21800 & 80 & \multirow{5}{*}{8} & \multirow{5}{*}{$\mathrm{mPWM}$} & \multirow{5}{*}{$\mathrm{M}=5, \mathrm{r}=0.25, \mathrm{k}=1$} & \multirow{5}{*}{12} & 90.78 & 86.29 & 95.27 \\
\hline & 23754 & 95 & & & & & 92.18 & 88.24 & 96.12 \\
\hline & 23142 & 90 & & & & & 91.69 & 87.82 & 95.57 \\
\hline & 24270 & 100 & & & & & 91.53 & 87.36 & 95.71 \\
\hline & 25122 & 110 & & & & & 91.98 & 87.95 & 96.01 \\
\hline
\end{tabular}

prediction using the EEG benchmark Epileptic Seizure Recognition dataset of $\mathrm{UCI}^{1}$. This dataset contains EEG signals of length 23.6 seconds from 500 different individuals. Then, these EEG signals are framed using sliding windows of length 1 second to reconstruct $23 \times 500=11500$ frames of data (row), each frame contains 178 sampling-points for 1 second(column), the last column represents the label $y=\{1,2,3,4,5\}$ [25].

${ }^{1}$ url: https://archive.ics.uci.edu/ml/datasets/Epileptic+Seizure+Recognition
For epileptic seizure binary classification, Class 0 represent subjects falling in classes $2,3,4$, and 5 . The positive class is represented by all subjects falling in class 1 . As the dataset will be unbalanced, we randomly selected a balanced dataset and applied 5-folds cross-validation. The obtained results attain a good prediction of up to $96 \%$. The operation is repeated 10 times in order to study the robustness of the classification model. The average performance of the 10 experiments is 
TABLE VIII: Classification performance using different classifiers $[L=95$, step $=8, M=6, r=0.33]$.

\begin{tabular}{|c|c|c|c|c|c|c|c|}
\hline Classifier & KNN & Linear SVM & RBF SVM & Decision Tree & Random Forest & MLP & NB \\
\hline Parameters & $K=5$ & $\begin{array}{l}\text { kernel="linear" } \\
C=0.025\end{array}$ & $\begin{array}{l}\text { kernel="rbf" } \\
\text { gamma=2 } \\
C=1\end{array}$ & max_depth=5 & $\begin{array}{l}\text { max_depth=5 } \\
\text { n_estimators=10 } \\
\text { max_features=1 }\end{array}$ & alpha=1 & defaults \\
\hline Accuracy & 92.32 & 79.10 & $\mathbf{9 2 . 4 8}$ & 85.30 & 84.03 & 80.78 & 69.42 \\
\hline Sensitivity & 95.93 & 91.01 & $\mathbf{9 7 . 7 6}$ & 91.98 & 91.06 & 87.14 & 68.19 \\
\hline Specificity & 88.70 & 67.20 & $\mathbf{8 7 . 2 0}$ & 78.62 & 77.01 & 74.42 & 70.64 \\
\hline Precision & 95.62 & 88.21 & $\mathbf{9 7 . 5 0}$ & 90.75 & 89.60 & 85.57 & 69.07 \\
\hline Recall & 88.70 & 67.20 & $\mathbf{8 7 . 2 0}$ & 78.62 & 77.01 & 74.42 & 70.64 \\
\hline F1-score & 92.03 & 76.27 & $\mathbf{9 2 . 0 6}$ & 84.25 & 82.81 & 79.47 & 69.81 \\
\hline ROC-AUC & 0.92 & 0.79 & $\mathbf{0 . 9 2}$ & 0.85 & 0.84 & 0.81 & 0.69 \\
\hline
\end{tabular}

TABLE IX: The 5-Folds cross-validation classification using QuPWM with time and frequency domain for EEG classification from the UCI dataset.

\begin{tabular}{|c|c|c|c|c|c|c|}
\hline QuPWM & Domain & Parameters & Feature Size & Accuracy & Sensitivity & Specificity \\
\hline \multirow{2}{*}{ PMW } & Time & $\mathrm{M}=8, \mathrm{r}=1$ & 2 & 94.38 & 92.05 & 96.72 \\
\cline { 2 - 7 } & Frequency & $\mathrm{M}=6, \mathrm{r}=0.6$ & 2 & 95.71 & 94.97 & 96.44 \\
\hline \multirow{2}{*}{ mPWM } & Time & $\mathrm{M}=10, \mathrm{r}=0.6,, \mathrm{k}=1$ & 20 & 94.78 & 93.58 & 95.98 \\
\cline { 2 - 7 } & Frequency & $\mathbf{M = 6}, \mathbf{r = 0 . 6}, \mathbf{k = 1}$ & $\mathbf{1 6}$ & $\mathbf{9 6 . 1 6}$ & $\mathbf{9 5 . 5 5}$ & $\mathbf{9 6 . 7 6}$ \\
\hline
\end{tabular}

reported in table IX.

\section{E. The main limitations of the QuPWM features}

The above results show that the proposed QuPWM method is able to extract valuable features and significantly reduce the feature size. However, this method has the following limitations which will be handled in future works:

- QuPWM is more suitable for balanced dataset classification. In the case of highly unbalanced datasets, the samples used to build the PWM matrices should be carefully chosen. For instance, clustering techniques such as k-mean may be used to overcome this challenge.

- In case of a big dataset $\left(>10^{4}\right.$ samples and $>10^{3}$ samples-points ), the PWM matrices will be bigger and more sparse, which needs more memory and high-speed processors. Therefore, compressing sensing techniques or Principle Component Analysis (PCA) can be used to compress the matrices while keeping similar performance.

\section{CONCLUSIONS}

We developed a feature extraction method, called QuPWM, for epileptic spikes classification in MEG and EEG signals. This method is based on combining the position weight matrix (PWM) method with digital quantization. The method shows great potential in improving the spike classification accuracy, specifically when using the frequency domain through a ShortTime Fourier Transform of raw input signals. The method is validated using three different classification experiments using MEG and EEG signals. For instance, the QuPWM feature could achieve up to $88 \%$ in sensitivity and $97 \%$ in specificity for a dataset consisting of nine epileptic patients using the MEG signal. Besides, it achieves up to $95 \%$ in sensitivity and $96 \%$ in specificity using the EEG dataset. In the future, we are clustering the input sequences to build PWM-specific feature for each cluster of similar subjects, to build a hybrid detection model which might improve the accuracy, especially for outliers samples.

\section{ACKNOWLEDGMENT}

Research reported in this publication was supported by King Abdullah University of Science and Technology (KAUST) 
in collaboration with King Abdulaziz City for Science and Technology (KACST) and King Saud University (KSU).

\section{FUNDING}

This research project has been funded by King Abdullah University of Science and Technology (KAUST) Base Research Fund (BAS/1/1627-01-01), in collaboration with King Abdulaziz City for Science and Technology (KACST) and King Saud University (Deanship of Scientific Research, Grant: RG-1440-112).

\section{REFERENCES}

[1] R. S. Fisher, W. v. E. Boas, W. Blume, C. Elger, P. Genton, P. Lee, and J. Engel Jr., "Epileptic seizures and epilepsy: Definitions proposed by the international league against epilepsy (ilae) and the international bureau for epilepsy (ibe)," Epilepsia, vol. 46, no. 4, pp. 470-472, 2005. [Online]. Available: https://onlinelibrary.wiley.com/doi/ abs/10.1111/j.0013-9580.2005.66104.x

[2] M. Hämäläinen, R. Hari, R. J. Ilmoniemi, J. Knuutila, and O. V. Lounasmaa, "Magnetoencephalography-theory, instrumentation, and applications to noninvasive studies of the working human brain," Reviews of Modern Physics, vol. 65, no. 2, pp. 413-497, apr 1993. [Online]. Available: https://link.aps.org/doi/10.1103/RevModPhys.65. 413

[3] H. Stefan and E. Trinka, "Magnetoencephalography (MEG): Past, current and future perspectives for improved differentiation and treatment of epilepsies," Seizure, vol. 44, pp. 121-124, jan 2017. [Online]. Available: https://www.sciencedirect.com/science/article/pii/ S1059131116302217?via\{\\%\}3Dihub

[4] D. J. Englot, S. S. Nagarajan, B. S. Imber, K. P. Raygor, S. M. Honma, D. Mizuiri, M. Mantle, R. C. Knowlton, H. E. Kirsch, and E. F. Chang, "Epileptogenic zone localization using magnetoencephalography predicts seizure freedom in epilepsy surgery," Epilepsia, vol. 56, no. 6, pp. 949-958, jun 2015. [Online]. Available: https://www.ncbi.nlm.nih.gov/pubmed/25921215https://www. ncbi.nlm.nih.gov/pmc/PMC4457690/

[5] T. B. Bates, Human brain $3 D$ model stock image, Apr 2012. [Online]. Available: https://depositphotos.com/9813537/ stock-photo-human-brain-3d-model.html

[6] Alexmit, Epileptic seizures and depression may share a common genetic cause, study suggests, Jan 2018. [Online]. Available: https://medicalxpress.com/news/ 2018-01-epileptic-seizures-depression-common-genetic.html

[7] S. Baillet, "Magnetoencephalography for brain electrophysiology and imaging," Nature Neuroscience, vol. 20, p. 327, feb 2017. [Online]. Available: https://doi.org/10.1038/nn.4504http://10.0.4.14/nn.4504

[8] F. E. Abd El-Samie, T. N. Alotaiby, M. I. Khalid, S. A. Alshebeili, and S. A. Aldosari, "A Review of EEG and MEG Epileptic Spike Detection Algorithms," IEEE Access, vol. 6, pp. 60 673-60 688, 2018.

[9] A. Ossadtchi, S. Baillet, J. C. Mosher, D. Thyerlei, W. Sutherling, and R. M. Leahy, "Automated interictal spike detection and source localization in magnetoencephalography using independent components analysis and spatio-temporal clustering," Clinical Neurophysiology, vol. 115 , no. 3 , pp. 508-522, 2004. [Online]. Available: http: //www.sciencedirect.com/science/article/pii/S138824570300405X

[10] M. I. Khalid, T. Alotaiby, S. A. Aldosari, S. A. Alshebeili, M. H. Al-Hameed, F. S. Y. Almohammed, and T. S. Alotaibi, "Epileptic MEG Spikes Detection Using Common Spatial Patterns and Linear Discriminant Analysis," IEEE Access, vol. 4, pp. 4629-4634, 2016.

[11] M. I. Khalid, T. N. Alotaiby, S. A. Aldosari, S. A. Alshebeili, M. H. Alhameed, and V. Poghosyan, "Epileptic MEG Spikes Detection Using Amplitude Thresholding and Dynamic Time Warping," IEEE Access, vol. 5, pp. $11658-11667,2017$.

[12] A. Chahid, T. N. Alotaiby, S. A. Alshebeili, and T.-M. Laleg-Kirati, "Feature Generation and Dimensionality Reduction using the Discrete Spectrum of the Schrödinger Operator for Epileptic Spikes Detection," in 201941 st Annual International Conference of the IEEE Engineering in Medicine and Biology Society (EMBC), 2019.

[13] T.-M. Laleg-Kirati, E. Crépeau, and M. Sorine, "Semi-classical signal analysis," Mathematics of Control, Signals, and Systems, vol. 25, no. 1, pp. 37-61, 2013. [Online]. Available: http://dx.doi.org/10.1007/ s00498-012-0091-1
[14] S. Taulu and J. Simola, "Spatiotemporal signal space separation method for rejecting nearby interference in MEG measurements," Physics in Medicine and Biology, vol. 51, no. 7, pp. 1759-1768, mar 2006. [Online]. Available: https://doi.org/10.1088\%2F0031-9155\% 2F51\%2F7\%2F008

[15] R. M. Gray and D. L. Neuhoff, "Quantization," IEEE Transactions on Information Theory, pp. 2325-2383, 1998.

[16] D. Pollard, "Quantization and the method of k-means," IEEE Transactions on Information theory, vol. 28, no. 2, pp. 199-205, 1982.

[17] M. Nikulin, "Three-sigma rule," Encyclopedia of Mathematics, available at: http://www. encyclopediaofmath. org/index. php, 2011.

[18] F. Pukelsheim, "The three sigma rule," The American Statistician, vol. 48, no. 2, pp. 88-91, 1994. [Online]. Available: https: //amstat.tandfonline.com/doi/abs/10.1080/00031305.1994.10476030

[19] G. D. Stormo, T. D. Schneider, L. Gold, and A. Ehrenfeucht, "Use of the perceptronalgorithm to distinguish translational initiation sites in e. coli," Nucleic acids research, vol. 10, no. 9, pp. 2997-3011, 1982.

[20] G. Z. Hertz and G. D. Stormo, "Identifying dna and protein patterns with statistically significant alignments of multiple sequences." Bioinformatics (Oxford, England), vol. 15, no. 7, pp. 563-577, 1999.

[21] R. Staden, "Computer methods to locate signals in nucleic acid sequences," 1984.

[22] M. N. Akhtar, S. A. Bukhari, Z. Fazal, R. Qamar, and I. A. Shahmuradov, "POLYAR, a new computer program for prediction of poly (A) sites in human sequences," BMC genomics, vol. 11, no. 1, p. 646, 2010.

[23] J. E. Tabaska and M. Q. Zhang, "Detection of polyadenylation signals in human DNA sequences," Gene, vol. 231, no. 1, pp. 77-86, 1999. [Online]. Available: http://www.sciencedirect.com/science/article/ pii/S0378111999001043

[24] A. Chahid, T. N. Alotaiby, and L.-K. Alshebeili, Saleh A. Taous-Meriem, "Position weight matrix, gibbs sampler, and the associated significance tests in motif characterization and prediction," Scientifica, vol. 2012, 2012.

[25] R. G. Andrzejak, K. Lehnertz, F. Mormann, C. Rieke, P. David, and C. E. Elger, "Indications of nonlinear deterministic and finite-dimensional structures in time series of brain electrical activity: Dependence on recording region and brain state," Phys. Rev. E, vol. 64, p. 061907, Nov 2001. [Online]. Available: https://link.aps.org/doi/10.1103/PhysRevE.64.061907

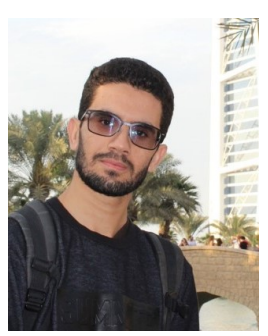

Abderrazak Chahid received the B.Sc. in Electrical Engineering and Power Electronics from Sultan Moulay Slimane University. He then got an M.Sc. degree in Electrical Engineering from the National School of Applied Sciences in Morocco before obtaining another M.Sc. degree of science in Embedded Systems from Lorraine University in France. Actually, he is a Ph.D. candidate in Electrical engineering department within CEMSE division in King Abdullah University of Science and Technology (KAUST) His current research interest is signal processing-based features generation for biomedical signal classification and prediction.

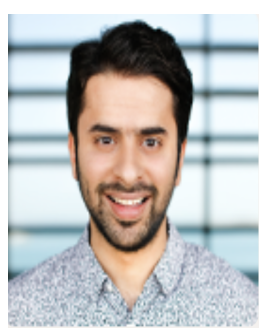

Fahad Albalawi Fahad Albalawi received his B.S. in 2008 in Electrical Engineering from Umm Alqura University, Makkah. He received his M.S. degree in 2013 in Electrical Engineering from George Washington University, Washington DC. In June 2017, Fahad obtained his Ph.D. degree in Electrical Engineering from University of California, Los Angeles. Right after his $\mathrm{PhD}$ graduation, Fahad obtained an assistant professor position of Electrical Engineering at Taif University, Saudi Arabia. His research interests include model predictive control, nonlinear systems, , dynamic process optimization, process operational safety and machine learning. 


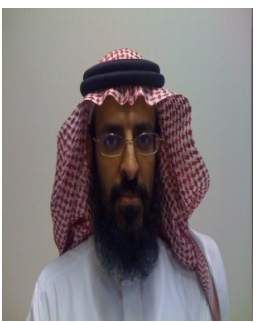

Turky Nayef Alotaiby received the B.Sc. degree in computer information sciences from King Saud University (KSU), Saudi Arabia, in 1992, the M.Sc degree in computer information systems from Florida Institute of technology, USA, 1998, and the $\mathrm{PhD}$. Degree in computer science from Florida Institute of Technology, USA, 2004. He spent three months (scientific trip) in Montreal Neurological Institute, McGill University, Canada, 2013. Currently, he is an associate research professor in the National Center for Artificial Intelligence and Big Data Technology/ Communication and Information Technology Institute, King Abdul-Aziz City for Science and Technology (KACST), Saudi Arabia. Dr. Alotaiby published several papers in conferences and refereed journals. His current research interests include physiological signals processing, pattern recognition, and time series modeling and prediction

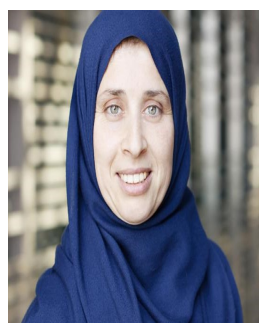

Taous-Meriem Laleg-Kirati is an Associate Professor in the division of Computer, Electrical and Mathematical Sciences and Engineering at KAUST. She joined KAUST in December 2010. From 2009 to 2010 , she was working as a permanent research scientist at the French Institute for research in Computer Sciences and Control Systems (INRIA) in Bordeaux. She earned her Ph.D. degree in applied mathematics from INRIA Paris, in 2008. She holds a master in control systems and signal processing from University Paris 11 in France. Her research interest includes modeling, estimation, and control of complex systems and signal/image analysis. She considers applications in engineering and biomedical fields. Webpage:http://emang.kaust.edu.sa.

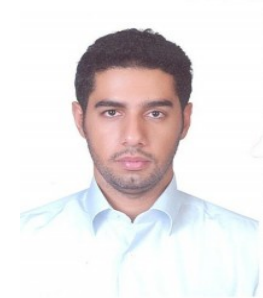

Majed Hamad Al-Hameed received the MBBS degree from King Saud University in 2000, then he went to do his basic Clinical Neurology Training with the University of Alberta, followed by one year in Basic Clinical Epilepsy and additional year in EEG and research with the University of Western Ontario in 2010. He is with King Fahad Medical City since 2011; interested in promotion of teaching and hold the position of Residency Program Director for neurology since 2012. Active in research and teaching. Active participant in Clinical Epilepsy with intraoperative and extraoperative brain mapping for epilepsy patients. Promote the epilepsy monitoring unit to increase the effectiveness of early surgical treatment in epilepsy in addition with brain modulation device. He holds the position of Adult Comprehensive Epilepsy Program Director and also, appointed as the Neurology Chairman in 2014.

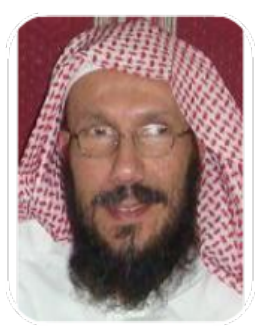

Saleh A. Alshebeili is professor and chairman (2001-2005) of Electrical Engineering Department, King Saud University. He has more than 25 years of teaching and research experience in the area of communications and signal processing. Dr Alshebeili is member of the board of directors of King Abdullah Institute for Research and Consulting Studies (KAIRCS, 2007-2009), member of the board of directors of Prince Sultan Advanced Technologies Research Institute (PSATRI, 2008-2017), the managing director of PSATRI (2008-2011), the director of SaudiTelecom Research Chair (2008-2012), and co-founder and director (2011Present) of the Technology Innovation Center, RF and Photonics for the e-Society (RFTONICS), funded by King Abdulaziz City for Science and Technology (KACST). Dr Alshebeili has been in the editorial board of Journal of Engineering Sciences of King Saud University (2009-2012). He has also an active involvement in the review process of a number of research journals, KACST general directorate grants programs, and national and international symposiums and conferences 